\title{
Cuidados Paliativos en Costa Rica
}

\section{Palliative Care in Costa Rica}

Carlos Acuña Aguilar ${ }^{1} \square$

\section{Resumen}

Filiación:

1.Universidad Santa Paula. San José, Costa Rica

Correspondencia: $\square$ Carlos Acuña Aguilar. cacuna@uspsantapaula.com

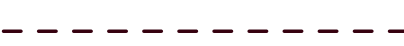

Forma de citar: Acuña Aguilar C. Cuidados paliativos en Costa Rica. Rev Ter. 2017;11(2):15-25.

Financiamiento:

Autofinanciado

Conflictos de Interés:

El autor declara no tener conflictos de interés en la presente publicación
En Costa Rica, durante las tres últimas décadas se ha logrado desarrollar diversos programas de cuidados paliativos tanto a nivel público como privado, dentro de la Seguridad Social como a través de organizaciones no gubernamentales sin fines de lucro, respectivamente; siendo considerada dentro del grupo de países con mayor desarrollo alcanzado en Latinoamérica. Se cuenta con legislatura en la materia desde el año 1994, mediante la resolución de un recurso de amparo presentado ante la Sala Constitucional, que garantiza el derecho a los Cuidados Paliativos para los habitantes del territorio nacional. Asimismo, se han aprobado otras leyes tales como: la Ley 8600 de beneficios para los responsables de pacientes en fase terminal, siendo el único país latinoamericano con esta legislatura. Además, desde el año 2008 existe un Consejo Nacional de Cuidados Paliativos, integrado por representantes titulares y suplentes de los actores sociales de organizaciones que tienen relación con los Cuidados Paliativos, y tiene como función asesorar al Estado en materia de Cuidados Paliativos. Este trabajo tiene como objetivo presentar el desarrollo histórico de los cuidados paliativos en Costa Rica.

Palabras claves: cuidados paliativos, medicina paliativa, legislación y jurisprudencia (Fuente: DeCS-BIREME)

\section{Abstract}

In Costa Rica, during the last three decades it has been possible to develop various palliative care programs, both public and private, within the Social Security as well as through non-profit non-governmental organizations, respectively; being considered within the group of countries with greater development reached in Latin America. There is a legislature on the matter since 1994, through the resolution of an appeal filed before the Constitutional Chamber, which guarantees the right to Palliative Care for the inhabitants of the national territory. Likewise, other laws have been approved such as: Law 8600 of benefits for those responsible for patients in the terminal phase, being the only Latin American country with this legislature. In addition, since 2008 there is a National Council of Palliative Care, composed of representatives and alternates of social actors of organizations that are related to Palliative Care, and its role is to 
advise the State on Palliative Care. The objective of this work is to present the historical development of palliative care in Costa Rica.

Keywords: palliative care, palliative medicine, legislation and jurisprudence (Source: MeSH-NLM)

\section{Introducción}

Los Cuidados Paliativos en Costa Rica inician a partir del año 1987, con la participación de la doctora Lisbeth Quesada Tristán, con estudios en Cuidados Paliativos realizados en los Estados Unidos de América, en el St Luke's Roosevelt Hospital Center de New York, quien se da a la tarea de difundir sus conocimientos en esta nueva rama de la medicina ${ }^{1}$ a través de cursos para profesionales en salud interesados, tanto dentro como fuera del sistema público de salud costarricense (Caja Costarricense de Seguro Social).

Posteriormente, empiezan a surgir una serie de iniciativas en el campo y es así como en el año 1990 en el Hospital Max Peralta de Cartago se forma un Comité de Cuidados Paliativos, que luego da origen en 1993 a la Asociación Caminemos Juntos (ASCAJU), cuyo propósito fundamental es la atención integral del enfermo en fase terminal de Cáncer y VIH-Sida de la población de la provincia de Cartago; siendo el primer grupo que nace formalmente en Costa Rica, como una organización no gubernamental sin fines de lucro. El 01 de octubre de 1990 la doctora Lisbeth Quesada Tristán junto con la doctora Ivette García y otros profesionales del Hospital Nacional de Niños, crean la primera Unidad de Cuidados Paliativos que atiende niños en el país; brindando sus servicios mediante los modelos de atención: intervención domiciliaria e intrahospitalaria. Esta Unidad realiza las Primeras Jornadas Médico Científicas en Cuidados Paliativos de Costa Rica y Centroamérica, en enero del año 1992, satisfaciendo la gran necesidad que imperaba en el país y la región, tanto en docencia como en conocimiento ${ }^{2}$.

Para el año 1991 se crea en el Hospital Calderón Guardia la Clínica del Dolor, por el Dr. Isaías Salas Herrera, que apoya a enfermos con dolor. En el año 1994 se constituye una Fundación que le brinda apoyo, soporte económico y logístico, posteriormente en el año 1995 incorpora los servicios de Cuidados Paliativos; en marzo de 1999 se transformó en un centro de referencia institucional, Ilamado Centro Nacional de Control del Dolor y Cuidados Paliativos (CNCDyCP), el cual en la actualidad cuenta con un grupo de unidades que constituyen una red a nivel institucional y un centro de docencia e investigación ${ }^{3}$.

En el año 1992 nace la Fundación Pro Unidad de Cuidados Paliativos, cuya razón de ser es apoyar a la Unidad de Cuidados Paliativos del Hospital Nacional de Niños, esta organización en el año 2000 crea el Albergue San Gabriel, ubicado en la ciudad de San José, el cual tiene un centro diurno de asistencia paliativa, servicios profesionales integrales, préstamo de equipo a domicilio, actividades recreativas, entre otros; en la actualidad esta organización se encuentra desarrollando un proyecto de construcción de la Nueva Casa de Cuidados Paliativos Pediátricos, con una infraestructura de ocho pisos. Asimismo, en el año 2007 se crea el Albergue Dr. Gastón Acosta Rúa, ubicado en el cantón de Pérez Zeledón en la zona sur de nuestro país, que brinda servicios de Cuidados Paliativos Pediátricos a la población infantil de esta región. La Unidad de Cuidados Paliativos del Hospital Nacional de Niños, con estas dos organizaciones brinda cobertura a todo el territorio nacional. En mayo del 2007 la Junta Directiva de la Caja Costarricense del Seguro Social realiza modificación-aclaración del artículo 21 de la sesión celebrada el 08 de abril de 1999, en la cual se establecen las diferencias entre Cuidados Paliativos de Adultos y Cuidados Paliativos Pediátricos, constituyéndose la Unidad de Cuidados Paliativos del Hospital Nacional de Niños, en el Centro Institucional de Cuidados Paliativos 


\section{Pediátricos ${ }^{2}$.}

En el año 1991 inicia sus funciones la Unidad de Cuidados Paliativos de Alajuela, operando inicialmente en las instalaciones del Hospital de Alajuela; en el año 1995 se crea la Asociación para la Atención Integral del paciente con Cáncer Terminal y/o Sida de Cuidados Paliativos de Alajuela, que apoya y administra esta Unidad. Cuenta en sus instalaciones desde el año 1993 con un Centro Diurno de Atención Paliativa, así como desde sus inicios con los modelos de atención de: consulta externa, visita domiciliar y consulta telefónica. A partir del año 2001 posee el modelo de atención tipo Hospicio (Hospicio Los Ángeles), constituyéndose en la única en el país que brinda este servicio.

Posteriormente han surgido otros grupos de Cuidados Paliativos en Hospitales de la Caja Costarricense del Seguro Social tales como: Hospital de San Ramón, Hospital de Heredia, Hospital Max Peralta en Cartago, Hospital de Turrialba, Hospital de Grecia, Hospital Raúl Blanco Cervantes, Hospital San Juan de Dios, Hospitales de Puntarenas, Liberia y Limón.

Mediante la formación de organizaciones no gubernamentales (ONG), sin fines de lucro, algunas comunidades se han organizado para brindar estos servicios, tales como: San Pedro de Poás, Barva, Alajuela, Cartago, San Joaquín de Flores, San Antonio de Belén en Heredia, La Unión en Cartago, Cuidad Quesada, Curridabat, entre otras ${ }^{2}$.

En la edición cartográfica del Atlas de Cuidados Paliativos en Latinoamérica, se manifiesta que a nivel nacional se cuenta con 43 equipos de Cuidados Paliativos multinivel correspondientes al $68 \%$ de los existentes al año 2013. Asimismo, se informa que en nuestro país con el uso de analgésicos opioides expresado en miligramos equivalentes de morfina por habitante, presenta una media de $9.6 \mathrm{mg} /$ habitante, superior a la media a nivel latinoamericano de 4.8 $\mathrm{mg} /$ habitante $^{5}$. Según el estudio realizado por la Asociación Latinoamericana de Cuidados Paliativos en el año 2014, fueron conformados tres grupos de avance en Cuidados Paliativos en Latinoamérica, según macro indicadores de desarrollo, ubicándose Costa Rica, Chile, México y Argentina en el grupo con más desarrollo; por otro lado, Bolivia, Honduras, República Dominicana y Guatemala se hallaron en el grupo de desarrollo inferior. Según el "Índex ALCP" Costa Rica registró el puntaje más elevado (8.1) entre los 20 países latinoamericanos evaluados ${ }^{6}$.

Costa Rica es uno de los países latinoamericanos que ha logrado legislar en materia de Cuidados Paliativos. Su historial legislativo en esta materia inicia en el año 1990 cuando se presenta ante la Sala Constitucional de la Corte Suprema de Justicia un Recurso de Amparo a favor de la señora Mónica Granados Chaverri, portadora de una enfermedad oncológica en fase terminal, a la cual su médico tratante le prescribe una dosis de morfina para control del dolor que es considerada por un profesional en Farmacia como muy elevada y no la despacha. Es así como la Sala Constitucional en la resolución del mismo expresa, que si bien es cierto que las farmacias son entes privados, realizan una función social directamente relacionada con la salud y no pueden negarse a despachar una receta legalmente constituida, además, un farmacéutico no puede negarse a despachar una receta legalmente constituida y no puede cambiarla. No puede invadir otro campo profesional y es responsabilidad exclusivamente del médico lo que le suceda al enfermo. Asimismo, la Sala Constitucional en la Sentencia 5130-94 estableció que del derecho a la vida que se establece en el Artículo 21 de la Constitución Política, donde se manifiesta que la vida es inviolable, se desprende el derecho a la salud y a los Cuidados Paliativos ${ }^{7}$.

Asimismo, en el documento entregado en el Primer Encuentro Nacional de Cuidados Paliativos celebrado 
el 05 de marzo del año 2007, se manifiesta que:

“......el derecho a la salud es un derecho derivado del derecho a la vida y la conectividad entre ellos es evidente. De manera más exacta podría decirse que el derecho a la salud es un objeto jurídico concreto del derecho a la vida; lo mismo ocurre cuando se refiere al derecho a la integridad física. El derecho a la salud tiene como propósito fundamental hacer efectivo el derecho a la vida, porque este no protege únicamente la existencia biológica de la persona, sino también los demás aspectos que de ella se derivan. La negación de prestar un servicio médico u hospitalario a una persona portadora de una enfermedad en fase terminal sea el servicio estatal o privado, atenta contra su derecho a la salud. Por ello, se debe aclarar que un acceso equitativo a los Cuidados Paliativos a todas las personas moribundas o en fase terminal es un derecho derivado del derecho a la salud, el cual contempla también la no denegación del tratamiento paliativo por falta de recursos económicos" ${ }^{8}$.

La Sala Constitucional de la Corte Suprema de Justicia costarricense, en la sentencia 1915-92 establece, que la persona portadora de enfermedad en fase terminal, es decir con un proceso irreversible, conserva el derecho a una muerte sin dolor y a una muerte digna. Con lo expuesto anteriormente la Sala Constitucional de la Corte Suprema de Justicia de Costa Rica, brinda una explicación del vínculo conceptual entre los Derechos Humanos y los Cuidados Paliativos, considerando que esos constituyen en sí mismos un derecho humano derivado del derecho a la vida y del derecho a una muerte digna ${ }^{9}$.

Con estas iniciativas se protege y se asegura el derecho a una muerte con dignidad y sin sufrimiento a las personas portadora de enfermedad en fase terminal y a toda la población que habita en el territorio costarricense; precedente que marca un antes y un después en la historia de los Cuidados Paliativos en nuestro país. Se crea un antecedente en que la población civil pueda optar por servicios en esta rama de la Medicina en los Sistemas de Salud nacionales existentes, tanto públicos como privados ${ }^{1}$.

El 25 de febrero del año 1998 se establece la Ley número 7756, llamada Beneficios para los responsables de las personas en fase terminal, publicada en el periódico oficial La Gaceta \# 56 del 20 marzo del mismo año, modificada por la Ley 8600 de setiembre del año 2007, publicada en el periódico oficial La Gaceta \# 192 del 05 de octubre del 2007, en la que se establece una licencia y subsidio para el cuidador principal de una persona en condiciones de terminalidad, que haya sido declarada por su médico tratante, por el tiempo que el enfermo amerite y con derecho a un subsidio establecido de la siguiente forma:

A. Hasta dos salarios base establecidos según la Ley $N^{\circ} 7337$, de 5 mayo de 1993, percibirán el cien por ciento $(100 \%)$ del promedio del ingreso.

B. Sobre el exceso de dos salarios y hasta tres salarios base establecidos según la Ley $N^{\circ} 7337$, de 5 mayo de 1993, percibirán el ochenta por ciento $(80 \%)$ del promedio del ingreso, por ese rango de salario.

C. Sobre el exceso de tres salarios base establecidos según la Ley $N^{0} 7337$, de 5 mayo de 1993 , percibirán el sesenta por ciento $(60 \%)$ del promedio del ingreso, por ese rango de salario ${ }^{10}$.

Convirtiéndose en el único país latinoamericano que cuenta con una legislación que beneficie a las personas que se constituyen en los cuidadores principales de estas personas enfermas; para optar por esta licencia los cuidadores deben ser cotizantes directos de la Caja Costarricense del Sistema de 
Seguridad Social, aportándoles un subsidio de acuerdo con su salario mensual y sin riesgo a perder su estabilidad laboral.

El 17 de abril del año 1998 se dicta la Ley 7765 de Creación del Instituto Costarricense del Cáncer, declarada inconstitucional el 30 de enero del 2008; en esta ley se reconoce y consolida la unión entre la Sociedad Civil y el Estado para atender en forma conjunta una problemática específica: la terminalidad de los enfermos con cáncer, unión que prevalece en la Ley 8718 titulada: Autorización para el cambio de nombre de la Junta de Protección Social y establecimiento de la distribución de rentas de las loterías nacionales, emitida el 17 de febrero del 2009, publicada en el periódico oficial La Gaceta número 154, del 10 de agosto del $2009^{11}$. De acuerdo con esta ley se redactó el Manual de Criterio para la distribución de recursos a las Asociaciones y Fundaciones que apoyan y administran Establecimientos de Cuidados Paliativos, según los artículos números 8 y 13 de esta ley ${ }^{12}$.

Mediante el Decreto Ejecutivo número 34560-S, del 08 de marzo del 2007, se realiza la oficialización de la Norma de Habilitación de los Establecimientos de Cuidados Paliativos y control del Dolor, emitida por el Ministerio de Salud como ente rector en la materia, en la cual se estipula los requisitos mínimos de deben cumplir estos establecimientos para su funcionamiento. Esta normativa fue revisada por el Consejo Nacional de Cuidados Paliativos en el año 2017, se encuentran en vías de oficialización y publicación en el Diario Oficial ${ }^{13}$.

Asimismo, también mediante Decreto Ejecutivo 34876-S, del 05 de diciembre del 2008, se crea el Consejo Nacional de Cuidados Paliativos, conformado por un cuerpo consultativo de expertos, representantes de los actores sociales en esta materia existentes en el país. Tiene como misión asesorar y coordinar en Cuidados Paliativos el despacho del Señor (a) Ministro (a) de Salud y los establecimientos de Cuidados Paliativos existentes, tanto públicos como privados con y sin fines de lucro. Este fue modificado en el Decreto Ejecutivo 36656$\mathrm{S}$, publicado en el Diario Oficial La Gaceta número 139 , el 19 julio de $2011^{14}$.

El Consejo Nacional de Cuidados Paliativos nace producto de un acuerdo consensuado tomado en el Encuentro Nacional de Establecimientos de Cuidados Paliativos celebrado en la ciudad de San José, en el año 2006, organizado por la Defensoría de los Habitantes y el Instituto Interamericano de Derechos Humanos. En esta actividad los actores sociales participantes representantes de los establecimientos que brindan atención en Cuidados Paliativos a nivel nacional, tanto públicos como privados, identifican la necesidad de que exista en nuestro país un cuerpo consultativo de expertos que le brinde información al Estado en materia de Cuidados Paliativos.

Su objetivo general es el de brindar asesoría al despacho ministerial como autoridad colegiada experta en el campo. En otras palabras, el Consejo Nacional de Cuidados Paliativos es el responsable mediante su cuerpo de expertos de asesorar al Ministerio de Salud, con el objeto de que este pueda ejercer las funciones rectoras en materia de Cuidados Paliativos.

El Consejo tendrá las siguientes funciones específicas:

A. Asesorar al Ministro (a) de Salud en los lineamientos técnicos y de política que deben ser considerados al formular el Plan Nacional de Cuidados Paliativos.

B. Asesorar al Ministro de Salud para que se logre la concertación y articulación de acciones entre las organizaciones públicas y privadas que se ocupan de los Cuidados Paliativos en el país. 
C. Apoyar al Ministerio de Salud para la instauración del Registro Estadístico Nacional de Enfermedades en condiciones paliativas atendidas por los establecimientos de Cuidados Paliativos, para su identificación, clasificación y selección.

D. Promover e impulsar que las instituciones y organizaciones no gubernamentales nacionales e internacionales asignen, suministren y financien, con la aportación de recursos y fondos, los proyectos o establecimientos de atención de Cuidados Paliativos.

E. Apoyar en la revisión de propuestas de proyectos de carácter normativo, financiero o social en materia de Cuidados Paliativos.

Está integrado por representantes titulares y suplentes de instituciones y organismos que dirigen, coordinan y financian los establecimientos de Cuidados Paliativos a nivel nacional, tales como: la Junta de Protección Social, Caja Costarricense de Seguro Social, Ministerio de Salud, Federación Costarricense de Cuidados Paliativos, Centro Nacional del Dolor y Cuidados Paliativos y un representante titular y suplente de las Fundaciones de Cuidados Paliativos. El Consejo nombra dentro de su seno un Presidente, un Vicepresidente y un Secretario, los integrantes no perciben dieta ni salario alguno por su cargo, son nombrados por un período de dos años y pueden ser reelectos. El Consejo se reunirá ordinariamente una vez al mes y extraordinariamente cuando lo considere necesario. Los aspectos operativos serán determinados en el Reglamento Interno acordado por los miembros del Consejo. Las sesiones serán convocadas por el Presidente o de oficio por el Secretario del Consejo, mediante comunicación escrita y con un mínimo de 12 horas de antelación, salvo cuando se trate de asuntos de urgencia donde no será necesaria la observancia de estas dos reglas. El Consejo se constituye únicamente para el cumplimiento e implementación del fin público que justifica la existencia del Órgano. Las donaciones que consisten en dinero que estén dirigidas a la concreción de las funciones y fines del Consejo ingresarán al Consejo Técnico de Asistencia Médico Social, órgano adscrito al despacho del Ministro de Salud según artículo 5 de la Ley Orgánica del Ministerio de Salud, y deberán ser presupuestados, gestionados y ejecutados en los términos en que dispone el ordenamiento jurídico.

El Ministerio de Salud, a través del Consejo Técnico de Asistencia Médico Social, para cumplir con las funciones asignadas al Consejo recibirá:

A. Subvenciones nacionales e internacionales, cuya finalidad exclusiva sea el funcionamiento del Consejo.

B. Contribuciones de las Instituciones Públicas del Estado que reciba el Ministerio de Salud, cuyo fin sea el mantenimiento del Consejo, de acuerdo con lo que establece la Ley Orgánica o Constitutiva de cada una de ellas.

C. Por legados, donaciones, herencias o subvenciones cuya finalidad sea apoyar las actividades, estudios y proyectos en materia de Cuidados Paliativos ${ }^{14}$.

La visión del Consejo es la de lograr Políticas Públicas y Nacionales en materia de Cuidados Paliativos que regulen los programas existentes a nivel nacional, que cumplan los principios y objetivos internacionales establecidos, con el objeto de brindar atención integral a las personas enfermas en condiciones paliativas y su grupo familiar, con solidaridad, igualdad, equidad, universalidad, calidad y seguridad ${ }^{2}$.

Para agosto del 2007 se oficializó el Plan Nacional de Cáncer para el período 2007-2016; en el año 2012 se publica el Plan Nacional para la Prevención y Control del Cáncer que comprende el periodo 2011-2017, en los cuales se contempla un apartado 
de Cuidados Paliativos ${ }^{15}$.

El 15 de setiembre de 2003 se crea la Federación Costarricense de Cuidados Paliativos, la cual agrupa a las organizaciones no gubernamentales sin fines de lucro cuya figura legal es una asociación. Según la Ley General de Asociaciones pueden constituirse asociaciones formadas por la reunión de dos o más asociaciones con personaría jurídica. La nueva entidad adquirirá personería jurídica independiente de la personería de las entidades que la componen. Esta forma de asociaciones se distinguirá con los términos de "federación", "liga" o "unión”, que deberán insertar en su nombre y que las asociaciones simples no podrán usar. Las asociaciones federadas pueden, a su vez, constituirse en las mismas condiciones en una nueva forma de asociación que llevará forzosamente el nombre de "confederación", término que se reserva exclusivamente para esta clase de entidades. La Federación Costarricense de Cuidados Paliativos no sólo tiene como finalidad representar a las asociaciones federadas ante las organizaciones públicas y privadas nacionales e internacionales, sino también asesorar, armonizar, apoyar y facilitar a sus asociados para el cumplimiento de los Principios y Objetivos Internacionales de los Cuidados Paliativos.

Dentro de los objetivos de la Federación Costarricense de Cuidados Paliativos están los siguientes:

- Reunir las Unidades de Cuidados Paliativos que son apoyadas por Asociaciones de Cuidados Paliativos existentes en el país.

- Promover acciones para la unificación de Organizaciones No Gubernamentales de Cuidados Paliativos

- Representary ser la voz de las Organizaciones No Gubernamentales de Cuidados Paliativos ante instituciones gubernamentales y privadas nacionales e internacionales.
- Servir de foro para la discusión y representación de la problemática nacional de las Organizaciones No Gubernamentales de Cuidados Paliativos.

- Promover, presentar e impulsar leyes que protejan y definan las normas y estatutos sobre Cuidados Paliativos, dándole asidero social, legal, médico y permanente

- Establecer convenios marco de cooperación con instituciones gubernamentales y no gubernamentales tanto nacionales como internacionales ${ }^{2}$.

La Federación Costarricense de Cuidados Paliativos ha participado en la confección del Manual de Normas de Habilitación y Acreditación del Ministerio de Salud brindando asesoría a la Junta de Protección para la elaboración del Manual de Distribución de fondos según la Ley 8718, en la redacción del proyecto de ley de Exoneración a las Asociaciones y Fundaciones de Cuidados Paliativos que generó la Ley 9140, comentada más adelante. También participó en la elaboración del Plan Nacional de Cáncer 2007; además de formar parte del Consejo Nacional Cuidados Paliativos con un representante titular y otro suplente ${ }^{2}$.

En la actualidad, por consenso de las organizaciones representadas ante la Federación Costarricense de Cuidados Paliativos, se trabaja en la elaboración de normas que regulen las funciones de miembros de las Juntas Directivas de las Asociaciones federadas, incluyendo responsabilidades y deberes, así como las exclusiones. Dentro de las funciones de la Juntas Directivas de las Asociaciones federadas se encuentra como prioritaria la de trabajar en función a un interés público y ser custodio del mismo, siendo los responsables de la organización ante la Sociedad Civil o la Comunidad y es función de la Federación velar por que ello se cumpla ${ }^{2}$. 
En el año 2005 se constituye la Asociación Costarricense de Medicina Paliativa y Medicina del Dolor, que asocia a los médicos con formación académica en Cuidados Paliativos, en el año 2007 esta organización se incorpora a la Federación Costarricense de Cuidados Paliativos. Entre sus principales logros ha sido que a través de su función como mediadora se logra incorporar la especialidad de Medicina Paliativa al Colegio de Médicos y Cirujanos de Costa Rica en el año 2008. Su función primordial radica en agrupar y representar a los médicos con especialidad en Medicina Paliativa, con Maestría en Cuidados Paliativos o egresados de la misma, así como médicos con especialidad en Medicina del Dolor; además de brindar educación continua mediante cursos, seminarios, simposios y congresos, tanto a nivel nacional como a nivel internacional ${ }^{2}$.

LaAsamblea Legislativa aprueba la Ley 9140 titulada: Exoneración a las Asociaciones y las Fundaciones que apoyan a las Unidades de Cuidados Paliativos, la cual es publicada en el periódico oficial La Gaceta Número 122 del 26 de junio del 2013. Esta ley manifiesta:

"Se exonera del pago de derechos e impuestos de inscripción sobre bienes inmuebles, excepto los impuestos municipales, a las asociaciones y fundaciones de Cuidados Paliativos que estén habilitadas y acreditadas por el Ministerio de Salud. Igualmente, se exonera de todo impuesto, tasas, sobretasas, derechos arancelarios, la importación compra local de vehículos destinados a uso exclusivo de dichas asociaciones y fundaciones, siempre y cuando estos bienes se destinen exclusivamente a la atención y el tratamiento directo de los pacientes atendidos por esas fundaciones. La exoneración que se otorgue en este artículo será para que estos bienes sean inscritos a nombre de la asociaciones y fundaciones de Cuidados
Paliativos; asimismo, no podrán ser enajenados a terceros hasta cumplido un plazo de diez años, desde su adquisición"16.

Esta ley es un logro para el desarrollo de los Cuidados Paliativos en nuestro país, en la actualidad existe un instructivo del Ministerio de Hacienda titulado: Gestión de Exención de Impuestos para Asociaciones y Fundaciones que Apoyan a las Unidades de Cuidados Paliativos, que garantiza la correcta aplicación de la ley ${ }^{17}$.

Aunque se ha logrado legislar en la materia, existe en la actualidad una necesidad país de contar con una ley exclusiva que garantice el derecho a los Cuidados Paliativos a los habitantes del territorio costarricense. Se han presentado ante la Asamblea Legislativa proyectos de ley con el objeto de llenar este vacío.

Después de 14 años de brindar los servicios de Cuidados Paliativos en Costa Rica, en el año 2003, se evidencia la necesidad de que los profesionales en Ciencias de la Salud cuenten con una formación universitaria en Cuidados Paliativos, que les brinde los conocimientos teórico prácticos especializados en esta materia. La Universidad Santa Paula diseña la malla curricular de la Maestría en Cuidados Paliativos, convirtiéndose en la primera organización a nivel nacional en brindar la educación universitaria formal en esta materia. Es así como en el año 2004 inicia en nuestro país la formación universitaria en Cuidados Paliativos, con grado académico de Maestría Profesional en esta universidad, con un postgrado teórico práctico dirigido a profesionales en ciencias de la salud, con una orientación interdisciplinaria, con elevado espíritu de servicio y gran compromiso hacia la persona enferma y su grupo familiar. Este brinda a los profesionales los conocimientos necesarios para una atención integral al enfermo y a su familia, con el objeto de que desarrollen la capacidad de realizar intervenciones en equipo, promoviendo el 
mejoramiento de la calidad de vida de las personas enfermas en condiciones paliativas, fomentando su dignidad, autonomía e independencia. Esta institución cuenta con dos Establecimientos en Cuidados Paliativos que funcionan como clínicas universitarias, dando soporte a los maestrantes en sus prácticas clínicas. Asimismo, se está diseñando la modalidad semipresencial del posgrado que será ofrecida a los países latinoamericanos ${ }^{18}$.

En el año 2006 la Universidad Católica inicia con la maestría en esta materia, también con grado académico de Maestría Profesional, constituyéndose en el segundo centro universitario en brindar esta carrera ${ }^{2}$.

En marzo de 2008, el Colegio de Médicos y Cirujanos de Costa Rica reconoce los requisitos para la inscripción de la especialidad en Medicina Paliativa. Publica en el periódico oficial La Gaceta la incorporación de la especialidad en Medicina Paliativa en Adultos y la especialidad en Medicina Paliativa en Niños; a partir de esta fecha se empiezan a inscribir al colegio profesional los profesionales en ciencias médicas que cumplen con los requisitos establecidos ${ }^{19}$.

En el año 2010 la Universidad de Costa Rica, en convenio con el Centro de Desarrollo Estratégico e Información en Salud y Seguridad Social (CENDEISSS), inicia la especialidad en Medicina Paliativa en adultos, impartida en los hospitales de la Caja Costarricense del Seguro Social, con doce créditos por semestre, con una duración de seis semestres, para un total de setenta y dos créditos $^{20}$. Con los siguientes objetivos: detectar, evaluar y atender médicamente las necesidades físicas, psicológicas, sociales, funcionales y espirituales de las personas con enfermedad incurable avanzada progresiva, potencialmente mortal, corto o mediano plazo. Así como, promover e implementar estrategias interdisciplinarias de cuidado del paciente con dolor, del paciente con enfermedad en fase terminal y su familia, destinadas a proporcionarles bienestar y calidad de vida hasta el final de la existencia y a guiarles durante el duelo familiar ${ }^{21}$.

En el 2018 se inauguran las nuevas instalaciones del Centro Nacional de Control del Dolor y Cuidados Paliativos, este proyecto consta de dos módulos de tres pisos, con una infraestructura de 5950 metros cuadrados. Las instalaciones están previstas para consultas médicas especializadas, procedimientos, docencia y capacitación, atención psicológica, atención social, nutricional, investigación, terapias de lenguaje, respiratoria y física, capilla, farmacia, toma de muestras de laboratorio, farmacia, entre otros ${ }^{4}$.

Finalmente, es importante destacar que a nivel de grado hay varias universidades que ofrecen cursos libres en materia de Cuidados Paliativos y en control del dolor; sin embargo, aún no se ha logrado incluirlos dentro de los planes de estudio de las carreras universitarias que forman profesionales en ciencias de la salud y que deberían incluir esta materia como parte de la formación integral de los profesionales de la salud.

\section{Referencias Bibliográficas}

1. Brenes $M$, Brenes J, Núñez X. Pasado y presente de los Cuidados Paliativos en Costa Rica. Rev Med Costa Rica Centroamérica. 2013;LXX(605):71-76

2. Acuña Aguilar C. Un modelo de Cuidados Paliativos en Costa Rica. San José, CR: Editorial Santa Paula; 2014.

3. Lopez De Behn E, Morales Alpizar C. Historia de los Cuidados Paliativos en Costa Rica dentro de la Seguridad Social. Med Paliat. 2005;12(2):9298.

4. Ministerio de Comunicación (CR). Mapa de obras 
Administración Solís Rivera [Internet]. San José, CR: Ministerio de Comunicación; 2018 [07 Marzo de 2018]. Centro Nacional de Control del Dolor y Cuidados Paliativos, San José. Disponible en: https://mapa.presidencia.go.cr/ccss/centro-nacional-de-control-de-dolor-y-cuidados-paliativos/

5. Pastrana T, De Lima L, Pons J, Centeno C. Atlas de Cuidados Paliativos en Latinoamérica. Edición Cartográfica 2013. Houston: IAHPCP Press; 2013.

6. Pastrana T, Tores Vigil I, De Lima L. Desarrollo de los Cuidados Paliativos en América Latina: Análisis mediante el uso de macroindicadores. Palliat Med. 2014;28(10):1231-1238.

7. Sentencia número 05130 de Sala Constitucional de la Corte Suprema de Justicia (CR), (07 Setiembre de 1994). Disponible en: https://vlex. co.cr/librariesprudencia-425

8. Quesada Tristán L. Derechos Humanos y Cuidados Paliativos. Rev Med Hondur. 2008; 76: 3943.

9. Jurisprudencia Constitucional. Derecho a morir con dignidad. Sentencia número 1915-92 de Sala Constitucional de la Corte Suprema de Justicia (CR). Rev Jurid Seguridad Soc. 1993;(3):54-63. Disponible en: http://www.binasss.sa.cr/revistas/ rjss/juridica3/07.pdf

10. Ley 8600. Modificación de la Ley $N^{0} 7756$, Beneficios para los responsables de pacientes en fase terminal. Diario Oficial La Gaceta (CR), 192, (05 de octubre de 2007). Disponible en: http://www.ilo.org/dyn/travail/docs/883/Ley\%20 No. $7756 \% 20$ Beneficios \%20para\%20los \%20 Responsables\%20de\%20Pacientes $\% 20$ en $\% 20$ Fase\%20Terminal.pdf

11. Ley 8718 Autorización para el cambio de nombre de la Junta de Protección Social y estableci- miento de la distribución de rentas de las loterías nacionales. Diario Oficial La Gaceta (CR), 154, (10 de agosto de 2009). Disponible en: http:// www.corteidh.or.cr/sitios/observaciones/2/XXII/ LEY\%208718\%20Junta\%20Proteccion\%20Social.doc.

12. Reforma Manual de Criterios para la Distribución de Recursos Ley 8718 (artículos 8 y 13 (Distribución de la utilidad neta de las loterías, juegos y otros productos de azar). Diario Oficial La Gaceta (CR), 86, (5 de mayo de 2011). Disponible en: http://www.pgrweb.go.cr/scij/Busqueda/Normativa/Normas/nrm_texto_completo.aspx?param1 $=$ NRTC \&nValor1=1\&nValor2=70196\&nValor3=84612\&strTipM=TC

13. Oficialización del Manual de normas para la habilitación de establecimientos que brindan atención en Cuidados Paliativos modalidad ambulatoria y domiciliar. No 34560 . Sistema Costarricense de Información Jurídica. (16 de mayo 2007). Disponible en: http://www.pgrweb.go.cr/ scij/Busqueda/Normativa/Normas/nrm_texto_ completo.aspx? param1=NRTC\&nValor1=1\&nValor2 $=63419 \&$ nValor3 $=72871 \&$ strTipM $=$ TC

14. Decreto Ejecutivo 36656-S. Diario Oficial La Gaceta (CR), 139, (19 de julio de 2011). Disponible en: http://www.gaceta.go.cr/pub/2011/07/19/ COMP_19_07_2011.pdf

15. Ministerio de Salud (CR). Plan Nacional para la Prevención y Control del Cáncer 2011-2017. San José, CR: Ministerio de Salud; 2012.

16. Ley 9140 Exoneración a las asociaciones y las fundaciones que apoyan a las Unidades de Cuidados Paliativos. Diario Oficial La Gaceta (CR), 122, (26 de junio del 2013). Disponible en: http://www.gaceta.go.cr/pub/2013/06/26/ ALCA116_26_06_2013.pdf 
17. Ministerio de Hacienda (CR). Gestión de exención de impuestos para asociaciones y fundaciones que apoyan a las unidades de cuidados paliativos [internet]. San José, CR: Ministerio de Hacienda; 2016. Disponible en: http://www. hacienda.go.cr/docs/577a8de0a848f_28\%20impuestos\%20para\%20cuidados\%20paliativos.pdf

18. Universidad Santa Paula, Departamento de Maestrías. Proyecto de Creación del Postgrado de Maestría en Cuidados Paliativos. Documento presentado a CONESUP. San José, CR: Universidad San Paula; 2003.

19. Normativa de maestría y doctorados académicos del Colegio de Médicos y Cirujanos de Costa Rica. Diario Oficial La Gaceta (CR), 50, (11 de marzo del 2008). Disponible en: https://www.imprentanacional.go.cr/gaceta/?date $=11 / 03 / 2008$

20. Centro de Desarrollo Estratégico e Información en Salud y Seguridad Social [Internet]. San José, CR: CENDEISSS; 2016. Posgrado. Disponible en: http://www.cendeisss.sa.cr/wp/index.php/ posgrado/

21. Universia Costa Rica [Internet]. Costa Rica: Universia. Especialidad en Medicina Paliativa, Universidad de Costa Rica. Disponible en: http:// www.universia.cr/estudios/universidad-costa-rica/especialidad-medicina-paliativa/st/237060 